\title{
OBSERVATIONS CONCERNANT L'EFFICACITÉ DE LA PÊCHE A L'ÉLECTRICITÉ DANS UNE RIVIÈRE DE LA ZONE A BARBEAU
}

\author{
par J.-C. PHILIPPART (1)
}

Chargé de Recherche du FNRS

\section{SOMMAIRE}

Introduction

1. Description de l'Ourthe et du tronçon étudié

2. Méthodes

2.1. Techniques de pêche à l'électricité

2.2. Mensurations, marquages et dénombrements statistiques

3. Résultats et discussion

3.1. Sélectivité en fonction de la taille
a) barbeau
b) chevaine, truite et autres espèces

3.2. Sélectivité en fonction des espèces
a) Réactivité intrinsèque des espèces au courant électrique
b) Taille moyenne spécifique
c) Mode de vie et habitat
d) Densité des populations

3.3. Efficacité absolue de la pêche en fonction du milieu
a) Influence de la profondeur (habitat)
b) Influence du débit
c) Influence d'autres facteurs

4. Conclusions

5. Références bibliographiques

(1) Institut de Zoologie de l'Université de Liège - Service d'Ethologie et Psychologie animales - Aquarium (Prof. J.C. RUNET) - Unité de Recherches Piscicoles, 22, quai Van Beneden, B - 4020 LIEGE, BELGIQUE. 


\section{RESUME}

Lors d'une étude quantitative des populations de poissons dans un tronçon de $9,6 \mathrm{~km}$ de l'Ourthe en Belgique, nous avons fait des observations méthodologiques concernant l'efficacité de la pêche à l'électricité dans une rivière de la Zone à Barbeau (largeur 25-30 m). Nous avons spécialement étudié la sélectivité en fonction de la taille et de l'espèce et l'influence des facteurs habitat (profondeur de l'eau) et débit et de quelques autres facteurs. Les résultats présentés dans cette étude permettent de mieux cerner les problèmes posés par le dénombrement des populations et communautés de poissons dans les rivières de moyenne importance.

\section{INTRODUCTION}

L'ètude quantitative des populations de poissons dans les grosses rivières de la Zone à Barbeau pose de nombreux problèmes méthodologiques. Les difficultés rencontrées proviennent d'abord des caractères particuliers de ces rivières : largeur supérieure à $20-25 \mathrm{~m}$, débit important surtout en dehors de l'étiage, hétérogénéité des habitats due à l'alternance de sections profondes et de zones à courant très rapide, développement parfois envahissant de la végétation aquatique. Par ailleurs, l'ichtyocénose des rivières à barbeau comprend plus de 20 espèces différentes par leur taille et leur écologie, ce qui rend difficile une étude quantitative sur l'ensemble de la communaute. Enfin, les trois espèces les plus caractéristiques de ce type de rivière - le barbeau, Barbus barbus (L.), le hotu Chondrostoma nasus (L.) et le chevaine, Leuciscus cephalus (L.) sont des Cyprinidae de grande taille, capables d'effectuer de longues migrations et, d'une manière générale, très mal connus quant aux aspects de l'écologie de leurs populations (stabilité spatiale, répartition selon les habitats, etc...) qui conditionnent les procédés d'échantillonnage et de dénombrement à adopter, les dimensions des secteurs à considérer, etc...

Lors d'une étude sur la dynamique et la production des populations de poissons de l'Ourthe (PHILIPPART, 1977a, 1977b), nous avons été confrontés à ces problèmes de stratégie d'échantilionnage; nous présentons dans cette note ulie série d'observations méthodologiques concernant l'influence du facteur espèce. de la taille des poissons, des caractères du milieu (profondeur, débit) sur l'efficacité absolue et la sélectivité de la pêche à l'électricité dans ce type de rivière.

\section{DESCRIPTION DE L'OURTHE ET DU TRONÇON ETUDIE}

L'Ourthe (fig. 1), principal affluent de la Meuse en Belgique, prend sa source sur le Plateau Ardennais et conflue avec le fleuve mosan à Liège après un parcours de $175 \mathrm{~km}$ (cf MICHA, 1971; PHILIPPART, 1977a). Le tronçon étudié est situé à environ $40 \mathrm{~km}$ de l'embouchure; il mesure une vingtaine de $\mathrm{km}$ et correspond à une Zone à Barbeau du type supérieur selon la classification de HUET (1949). Les principaux caractères morpho-dynamiques, physiques et chimiques du milieu sont présentés dans le tableau 1. La communauté ichtyologique comprend 23 espèces indigènes; l'ichtyomasse totale moyenne atteint $315 \mathrm{~kg} / \mathrm{Ha}$ et se répartit principalement entre le barbeau $(188 \mathrm{~kg} / \mathrm{Ha})$, le hotu $(51 \mathrm{~kg} / \mathrm{Ha})$, le chevaine $(21 \mathrm{~kg} / \mathrm{Ha})$, le goujon $(16 \mathrm{~kg} / \mathrm{Ha})$ et la truite $(11 \mathrm{~kg} / \mathrm{Ha})$.

Un profil longitudinal des profondeurs (fig. 2) établi sur une "zone centrale * de $9,6 \mathrm{~km}$ met en évidence la grande varièté des habitats et biotopes composant la rivière; il suggère en outre l'hétérogénéité de la répartition des pois- 


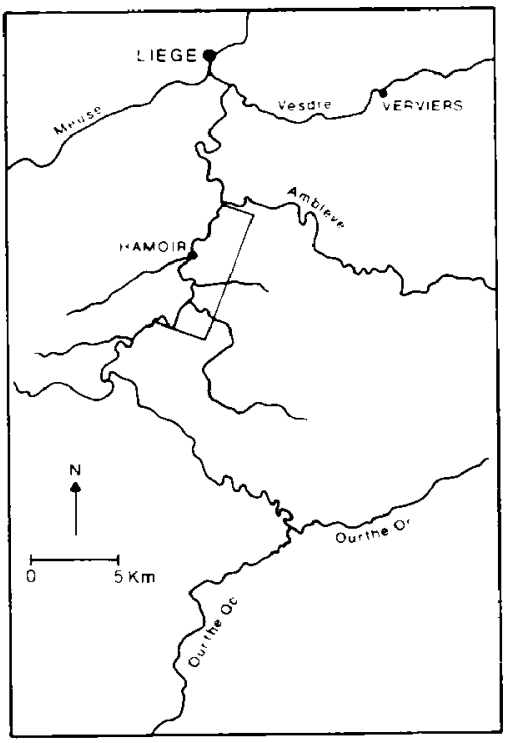

Fig. 1 : Cours de l'Ourthe et localisation du tronçon étudié.

sons, en nombre et en biomasse et partant la difficulté de réaliser des échantillonnages quantitatifs représentatifs de l'ensemble de la rivière et non des seuls habitats (berges, zones peu profondes, etc...) accessibles aux méthodes de pêche à l'èlectricité. Sur la base de critères physiographiques (profondeur, pente, largeur, vitesse du courant, facies lotique ou lentique), nous avons donc divisé le tronçon initial de $20,5 \mathrm{~km}$ et spécialement la zone centrale de $9,6 \mathrm{~km}$ en 40 sous-secteurs/ habitats (longueur moyenne $=220 \mathrm{~m}$ avec des extrêmes de 60 et $460 \mathrm{~m}$ ) plus ou moins homogènes et traités individuellement lors de tous les inventaires de population par pêche à l'électricité.

Tableau 1 : Principales caractéristiques morpho-dynamiques et physico-chimiques de l'Ourthe dans la région de Hamoir.

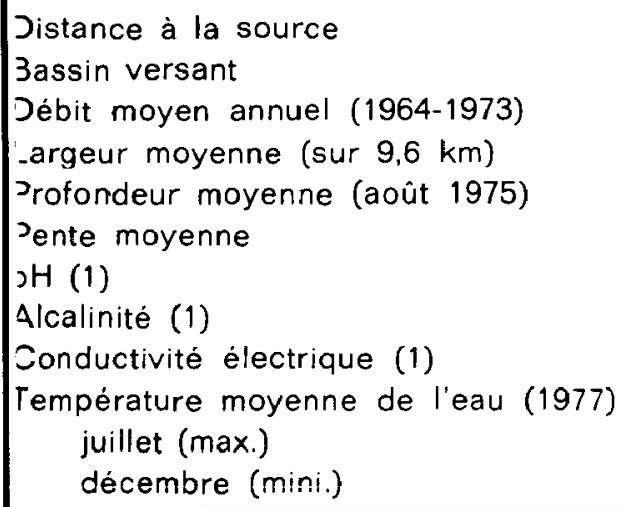

$\pm 140 \mathrm{~km}$

$1597 \mathrm{~km}^{2}$

$24 \mathrm{~m}^{3} / \mathrm{sec}$

$28,3 \mathrm{~m}(16-47)$

$0,46 \mathrm{~m}(0,13-1,54)$

$1,63 \%$

7,8

$92 \mathrm{mg} / \mathrm{l} \mathrm{CaCO}_{3}$

$255 \mu \mathrm{S} / \mathrm{cm}$

$17,6^{\circ} \mathrm{C}$

$4,4 \circ \mathrm{C}$

(1) Source : Dr I.P. DESCY, Institut de Botanique de IU.Lg. 


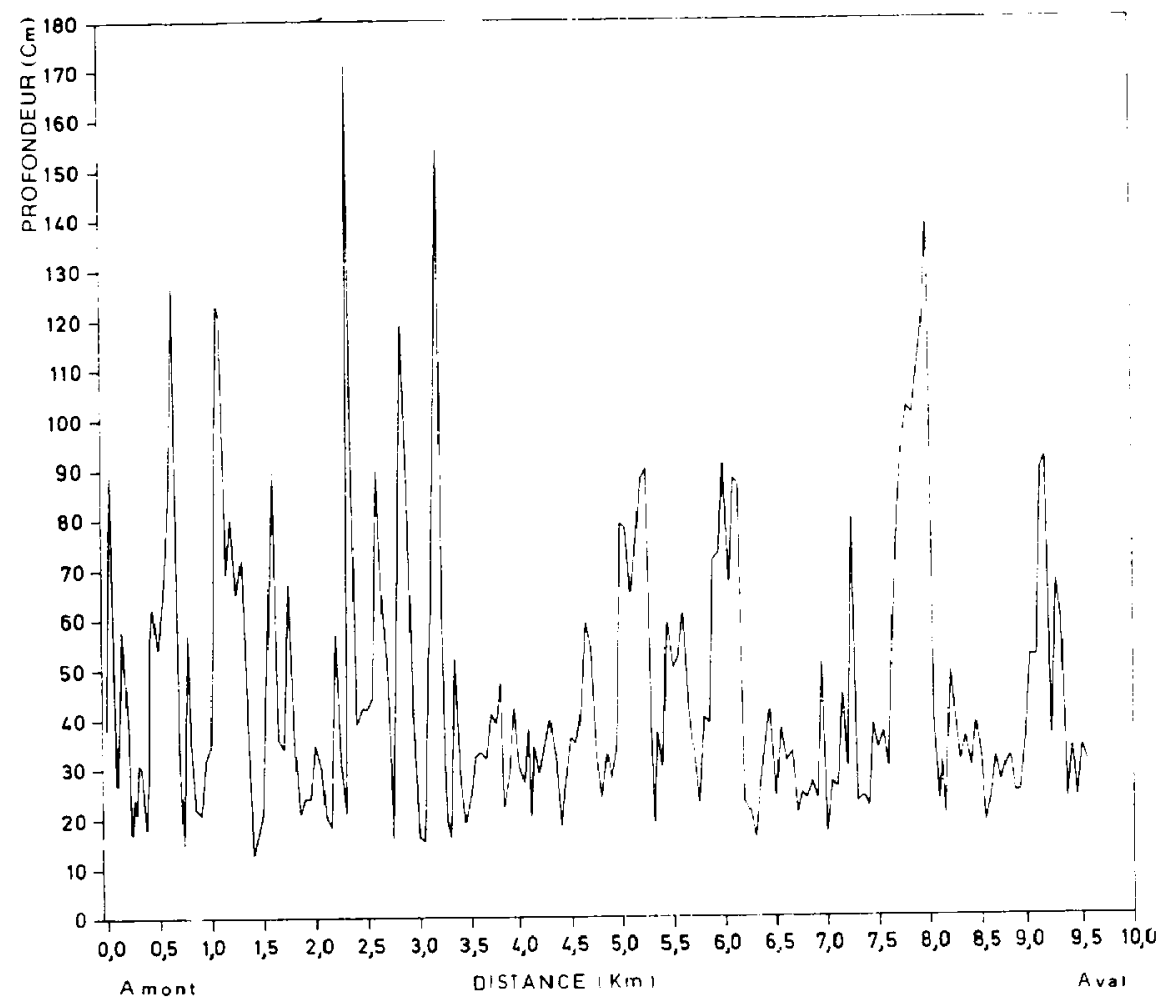

Fig. 2 : Profil longitudinal des profondeurs moyennes de l'Ourthe en août 1975 dans la zone centrale (profondeur moyenne par transect transversal tous les $50 \mathrm{~m}$ ).

\section{METHODES}

\subsection{Techniques de pêche}

Toute notre étude est basće sur des échantillonnages par pêche à l'électricité en courant continu $(220 \mathrm{~V})$ produit au moyen de générateurs Szabo de 0,8 et 1,4 KWA respectivement. Lors d'applications antérieures à l'Ourthe liégeoise (MICHA et RUWET, 1970; MICHA, 1971), le courant continu s'est avéré intéressant grâce à son pouvoir attractif à l'égard des Cyprinidae rhéophiles (surtout barbeau et chevaine) dans les herbiers à Renoncules en facies lotique et dans les abris naturels des berges en facies lentique.

En période de basses eaux $\left(<10 \mathrm{~m}^{3} / \mathrm{sec}\right)$ et pour l'échantillonnage de populations locales à forte densité (efforts successifs de capture. marquages intensifs, etc...), on pratique la pêche à l'électricité à la manière classique décrite par MICHA et RUWET (1970) : délimitation d'un secteur de 100-200 m et, à partir d'une base fixe de travail, balayage systématique de la rivière à contre-courant, en direction de la cathode immergée à l'amont. L'équipe de pêche comprend en moyenne 5 personnes : un porteur d'électrode, deux porteurs d'épuisette, un porteur de câble et un transporteur de poisson.

Pour des échantillonnages sur de grandes distances (expériences de marquage-recapture, étude des migrations) et en période de basses et moyennes eaux. on a progressivement substitué à la pêche électrique classique la pêche électrique 
à partir d'une base mobile (canot pneumatique). La riviere est praspectée à pied, dé l'amont vers l'aval et sur des distances de 500 à $1000 \mathrm{~m}$. Ce procédé permet un balayage systematique de toute la rivière, y compris dans des endroits peu ou pas accessibles par la route, et garantit en un minimum de temps un maximum de captures.

En période de hauies eaux et de manière genérale dans tous les endroits ou la profondeur dépas.je $1,50 \mathrm{~m}$, la pêche à l'électricité est pratiquee à partir d'ui canot spécialement aménagé (fig. 3) avec une équipe de 4-5 personnes; le cano: est dirigé à la rame ct exceptionnellement au moteur $(5 \mathrm{CV}$ ). Dans les conditions de trés fortes eaux (échantillonnage pendant la reproduction en marsavrii). on laisse le canot dériver p'us ou moins librement en freinant sa course autani que possible au moyen d'un poids (chaines; masse de $10 \mathrm{~kg}$ ).

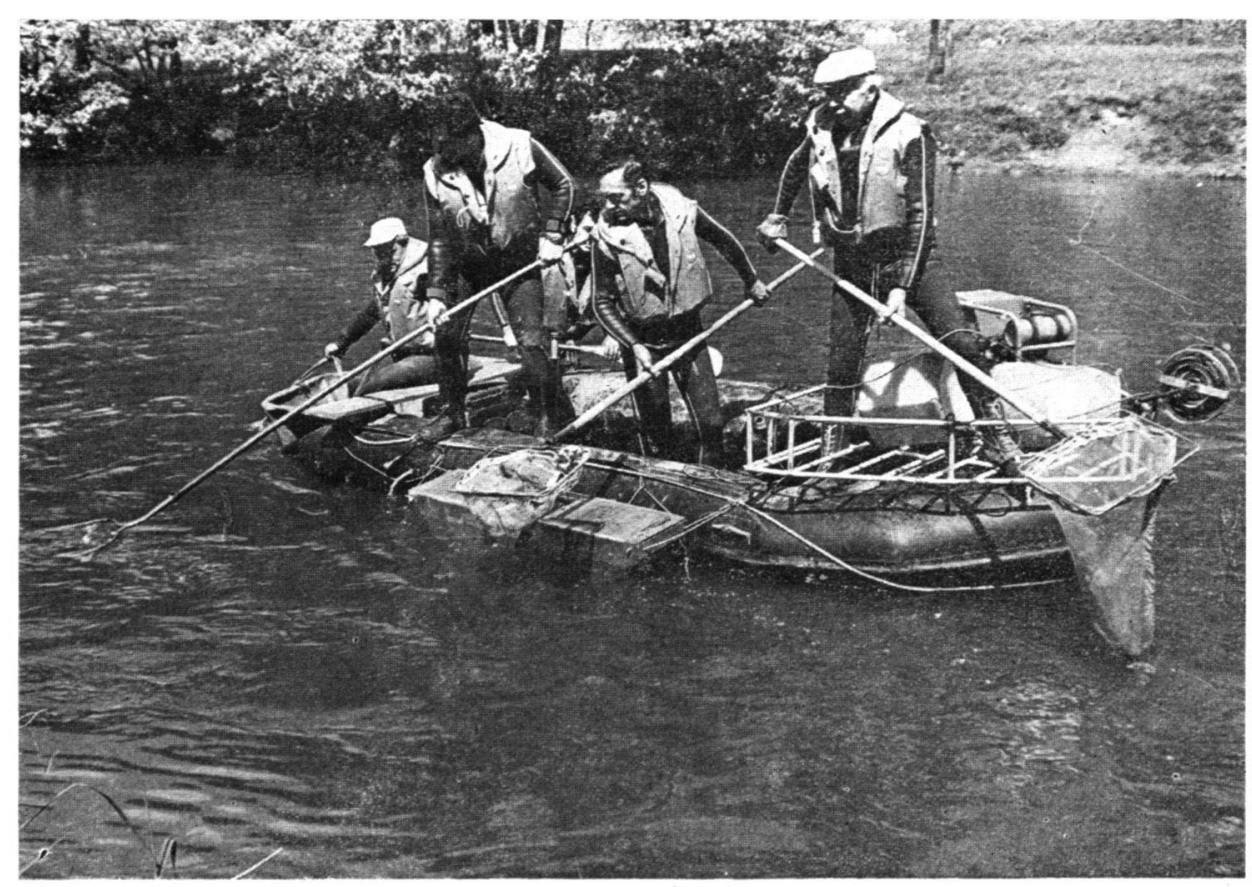

Fig. 3 : Pêche à l'électricité à partir d'un canot en période de fortes eaux et dans les zones profondes.

Des pêches à la senne et au filet maillant (MICHA et DE MOFFAERTS, 1975) ont été réalisées accessoirement pour la capture d'échantillons dans les zones profondes peu accessibles à la pêche à l'électricité; l'abondance de la végetation, les irrégularités du fond de la riviere (rocher, branches) et la vitesse du courant rendaient toujours la manœuvre ou le placement des filets très difficile. d'où un rendement faible et aléatoire.

Pour la prospection des grands fonds $(3-4 \mathrm{~m})$, on a utilise en quelques occasions la plongée en scaphandre autonome, avec capture des poissons à la main.

\subsection{Mensurations, marquages et dénombrements statistiques}

Après chaque pêche dans un sous-secteur/habitat donné. les poissons. préalablement anesthésiés au MS 222, étaient identifiés, mesurés (longueur au 
creux de la nageoire caudale), éventuellement pesés et soumis à un prélèvement d'écailles (détermination de l'âge-croissance) puis marqués et remis à l'eau.

Le marquage de groupe consistait en une mutilation de nageoire (surtout pelviennes et extrémités de la caudale) ou en un tatouage au moyen du "Fish Tatooing Panjet (colorant : bleu Durazol). Pour le marquage individuel, nous utilisions différents types de marques numérotées. Les meilleurs résultats furent obtenus chez le barbeau avec les marques ovales Présadom no 1 et 2 fixèes à la base du premier rayon épineux de la nageoire dorsale.

En 1971-1974, les populations ont été dénombrèes uniquement par les procédés classiques (cf SEBER, 1973) de capture-marquage et recapture simple (PETERSEN) et multiple (SCHNABEL; JOLLY-SEBER; Bailey triple catch).

\section{RESULTATS ET DISCUSSION}

L'efficacité de la pêche à l'électricité comme méthode d'échantillonnage quantitatif des populations de poissons a été testée lors d'expériences de marquage-recapture entreprises pour étudier les déplacements et les densités de peuplement. Les observations présentées ici concernent principalement le barbeau. Les données de base sont une série de statistiques de marquage-recapture dans différents secteurs de la rivière et au cours de plusieurs années (1972 à 1974).

L'efficacité de capture à l'égard d'un groupe prècis de poissons (ou en d'autres termes, sa probabilitè de capture) à un endroit et à un moment donnés est définie par la proportion $\mathrm{m} / \mathrm{N}$ du nombre $\mathrm{m}$ d'individus capturés par rapport à la population d'effectif total $N$. Les $m$ poissons étant marqués et remis à l'eau la proportion $\mathrm{m} / \mathrm{N}$ est normalement reflétèe - toutes autres conditions égales par la proportion $r / c$ du nombre $r$ d'individus marqués présents dans tout échantillon de c poissons prélevés quelque temps après le marquage. Nous analyserons successivement l'influence de la taille des poissons sur leur probabilité de capture (sélectivité de la méthode de pêche) ainsi que l'effet des conditions physiques du milieu sur l'efficacité absolue de la pêche.

\subsection{Sélectivité en fonction de la taille}

\section{a) Barbeau}

Comparons les valeurs des rapports $\mathrm{r} / \mathrm{c}$ pour 11 catégories de longueur (tableau 2, fig. 4). Le rapport $\mathrm{r} / \mathrm{c}$ passe de 0,105 dans la classe $125-150 \mathrm{~mm}$ à un maximum de \pm 0.200 dans la classe $200-275 \mathrm{~mm}$ pour diminuer ensuite dans les classes supérieures; un test de contingence $2 \times \mathrm{K}$ sur le nombre de marqués $r$ et de non marqués c-r révèle des différences significatives entre classes $\left(X^{2}=\right.$ 27,$84 ; 10$ d.I.). Cette hétérogénéité disparait pour l'ensemble des classes audessus de $175 \mathrm{~mm}\left(X^{2}=4,40 ; 8\right.$ d.l.; $\left.P=0,05\right)$; les variations du rapport $\mathrm{r} / \mathrm{c}$ qui se marquent à l'intérieur de ces classes sont dues au nombre de recaptures peu important au-delà de $35 \mathrm{~cm}$. 


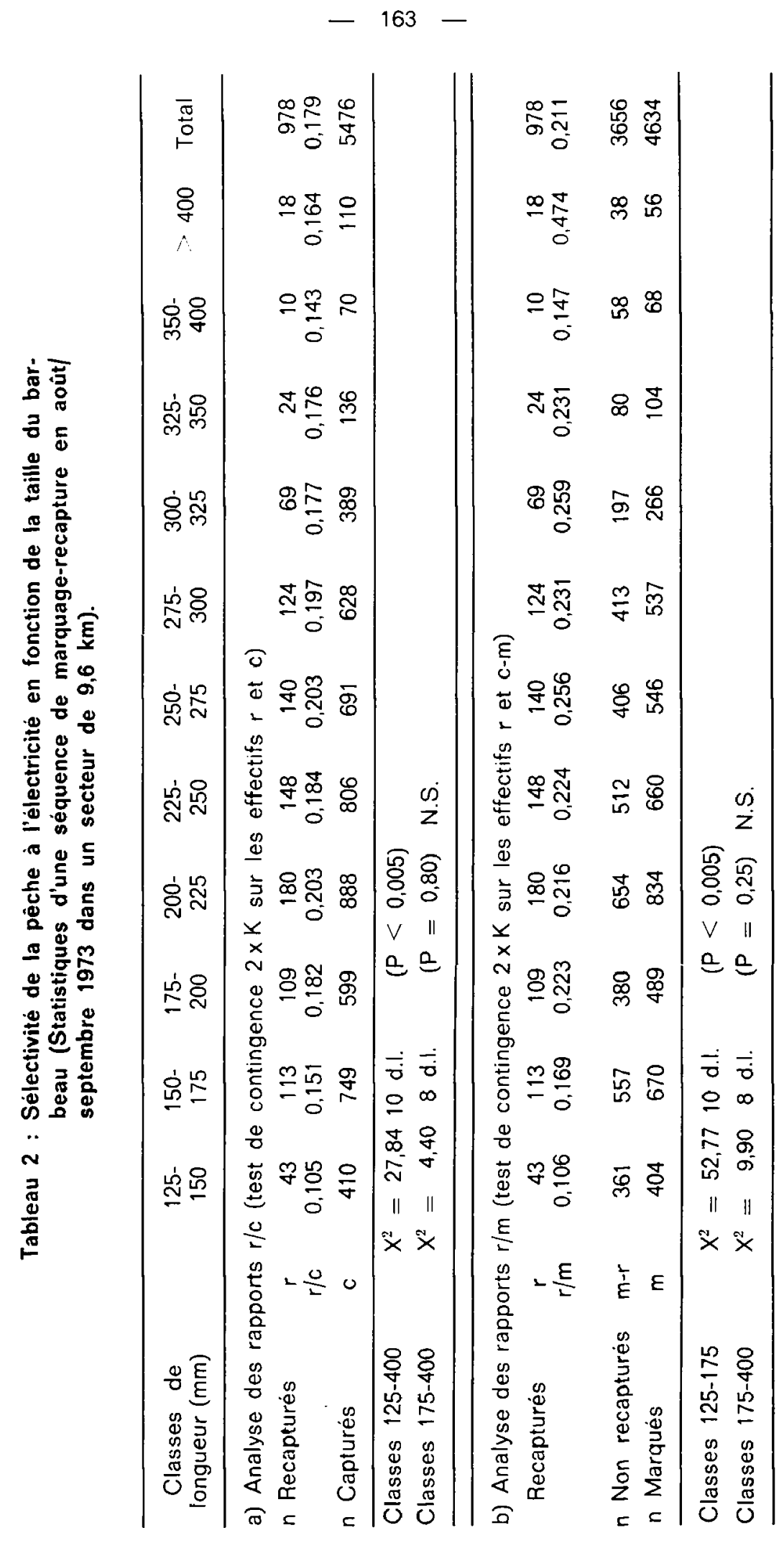




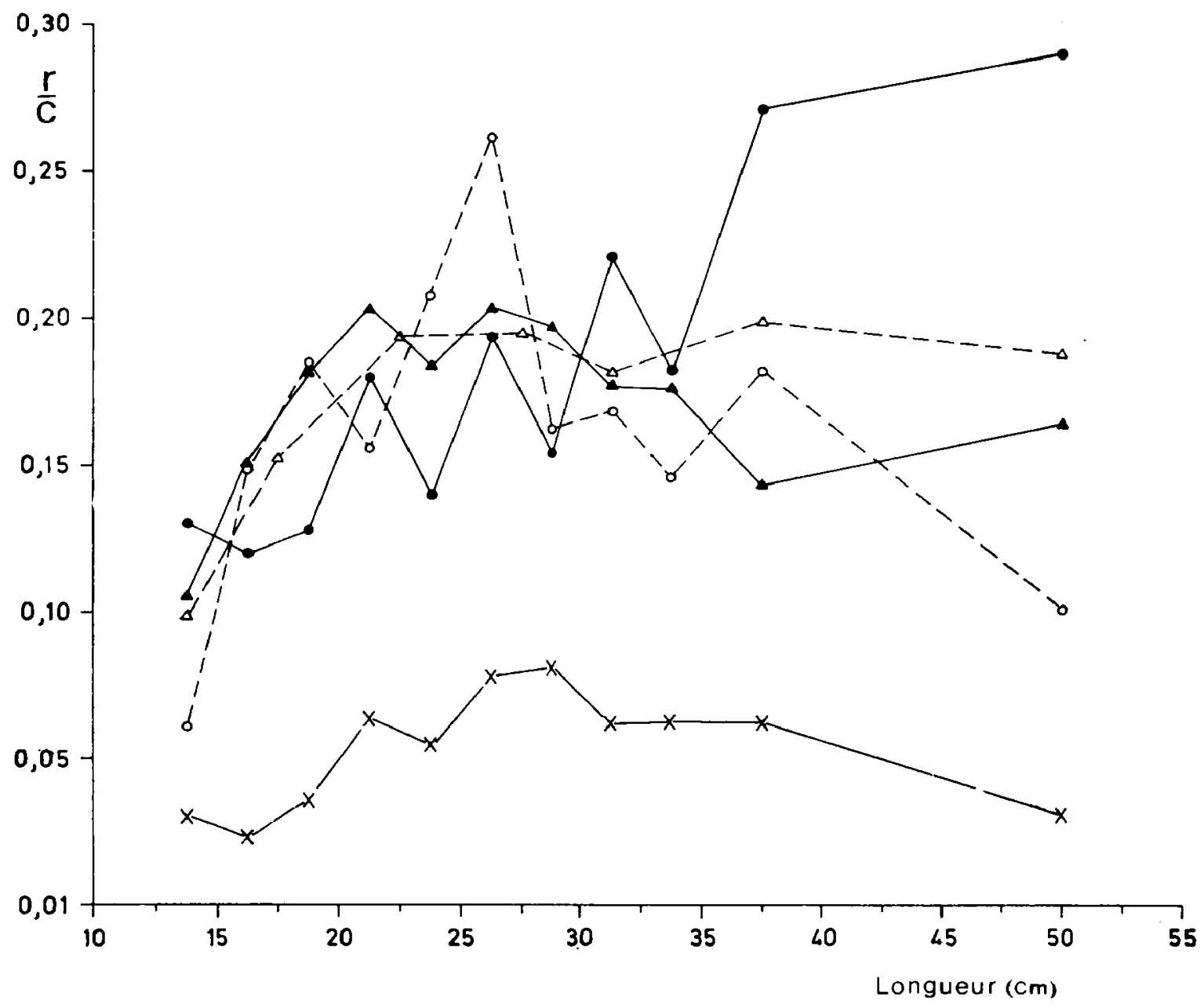

Fig. 4 : Courbes de sélectivité de la pêche à l'électricité selon la taille chez le barbeau. Les courbes sont établies à partir des rapports $\mathrm{r} / \mathrm{c}$ lors de séquences de marquage et recapture réalisées dans les 40 sous-secteurs/ habitats de la zone centrale à différentes dates et saisons et dans différentes conditions d'efficacité absolue de la pêche.
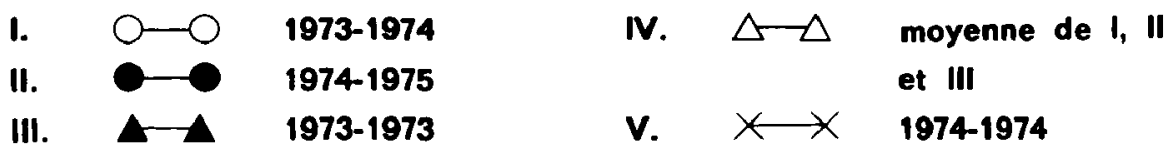

v. $\longleftrightarrow$ 1974-1974

Une analyse similaire des proportions de poissons marqués recapturés ultérieurement (rapports $\mathrm{r} / \mathrm{m}$ ) (tableau 2) conduit aux mêmes constatations. Chez le barbeau, la sélectivitè de la pêche à l'électricité correspond grosso modo à une officacité relative de capture qui decroit progressivement avec la taille pour les poissons mesurant moins de $20 \mathrm{~cm}$ (seuil de sélectivité). La sélectivité à l'égard de 
la taille varie dans le temps et dans l'espace en fonction des facteurs (hydrologiques, physiographiques) qui contrôlent l'efficacité absolue de capture. En général, plus l'efficacité de capture est faible, plus le seuil de sélectivité se relève (capture p!us aisée des grands poissons) (fig. 4).

\section{b) Chevaine, truite et autres especes}

Chez le chevaine et la truite, la sélectivité en fonction de la taille se traduit. comme chez le barbeau. par une courbe en cloche accroissement de l'efficacité de capture avec la taille (maximum pour les barbeaux et truites de $25-30 \mathrm{~cm}$ et pour les chevaines de $30.35 \mathrm{~cm}$ ) suivi d'une diminution pour les grands poissons, plus vifs à échapper à la capture ou concentrés dans les zones profondes difficilement pèchables (tableau 3).

Tableau 3 : Efficacité de la pèche à l'électricité selon les catégories de longueur chez trois poissons de l'Ourthe (l'efficacité de capture est mesurée par les rapports $r / c$ daris les expériences de dénombrement par marquage-recapture).

\begin{tabular}{|l|c|c|c|c|c|}
\hline \multirow{2}{*}{ Espèce } & \multicolumn{5}{|c|}{ Catégories de longueur (cm) } \\
\cline { 2 - 6 } & $15-20$ & $20-25$ & $25-30$ & $30-35$ & $>35$ \\
\hline Barbeau & 0,16 & 0,19 & 0,20 & 0,18 & 0,16 \\
Chevaine & $0,11(1)$ & - & 0,12 & 0,32 & 0,28 \\
Truite & 0,19 & 0,20 & 0,24 & $0,21(2)$ & - \\
\hline
\end{tabular}

(1) $18-25 \mathrm{~cm}$

(2) $>30 \mathrm{~cm}$

Pour les autres espèces (hotu, gardon, vandoise, ombre, perche), les faibles nombres de poissons capturès et recapturés ne permettent pas une analyse détaillèe de la sélectivité; à première vue, elle prend la même forme que dans les trois cas précédents.

\subsection{Sélectivitè en fonction des espèces}

Le tobleau 4 présente les statistiques de dénombrement par marquagerecapture simple (Petersen) de 10 espèces de poissons en 1973-74. sur un mème tronçon (zone centrale de $9,6 \mathrm{~km}-27,2 \mathrm{Ha}$ ). L'efficacité relative de capture mesurée par les rapports $\mathrm{r} / \mathrm{c}$ montre des variations spécifiquss très importantes: elle est maximale chez le chevaine, la truite et le barbeau, un peu plus faible chez "ombre et le hotu et très faible chez les autres espèces. Ces différences interspécifiques de capturabilité s'expliquent par l'influence combinée de 5 types de facteurs. 
Tableau 4 : Sélectivité de la pêche à l'électricitè en fonction des espèces dans l'Ourthe (efficacitè de capture mesurée par les rapports $\mathrm{r} / \mathrm{c}$ dans des expériences de dénombrement par marquage-recapture simple sur $9,6 \mathrm{~km}-27,2 \mathrm{Ha})$.

\begin{tabular}{|c|c|c|c|c|c|}
\hline \multirow{2}{*}{ Espèce } & \multirow{2}{*}{$\begin{array}{l}\text { Catégories } \\
\text { de longueur } \\
\text { (cm) }\end{array}$} & \multicolumn{3}{|c|}{ NOMBRE } & \multirow{2}{*}{$\begin{array}{c}\text { Rapport } \\
\mathrm{r} / \mathrm{c}\end{array}$} \\
\hline & & $\begin{array}{c}\text { marquès } \\
m\end{array}$ & $\begin{array}{c}\text { capturés } \\
\text { c }\end{array}$ & $\begin{array}{c}\text { recapturés } \\
r\end{array}$ & \\
\hline Barbeau & $>15$ & 4306 & 5016 & 931 & 0,19 \\
\hline Chevaine & $>18$ & $33 !$ & 322 & 69 & 0,21 \\
\hline Hotu & $>30(1)$ & 198 & 254 & 26 & 0.13 \\
\hline Vandoise & $>10$ & 142 & 234 & 3 & 0.01 \\
\hline Gardon & $>10$ & 144 & 120 & 8 & 0,07 \\
\hline Goujon (2) & $>10$ & 100 & 216 & 14 & 0,07 \\
\hline Truite & $>14$ & 659 & 727 & 145 & 0,20 \\
\hline Ombre & $>19$ & 92 & 122 & 19 & 0,16 \\
\hline Brochet & $>15$ & 37 & 78 & 5 & 0,06 \\
\hline Perche & $>10$ & 33 & 94 & 3 & 0,03 \\
\hline
\end{tabular}

(1) Population ne comprenant pas de poissons de $10-30 \mathrm{~cm}$

(2) Dénombrement dans un tronçon de $800 \mathrm{~m}$

\section{a) Réactivité intrinsèque des espèces au courant électrique}

Les espèces réagissent intrinsèquement de manière très différente au courant électrique. Se!on la conductivité de leur corps qui est liée au métabolisme (HALSBAND, 1967, on aurait donc le c'assement suivant des groupes d'esneces, par ordre decrossant de réactivite : salmonides $>$ cyprins d'sal vive > cyarins d'eau calme $>$ brochet et perche.

\section{b) Taille moyenne spécifique}

Ce facteur explique la faible capturabilité du goujon, de la vandoise ou du gardon par rapport aux cyprins de grande taille (barbeau, chevaine. hotu).

\section{c) Mode de vie et habitat}

Les espèces rhéophiles organisees en banc et à mode de vie plutôt pélagique, combre, hotu, vandoise) sont plus difficiles à pêcher que les espèces territoriales à répartition homogène (truite) ou que les espèces grégaires mais à mode de vie plutôt benthique (barbeau, goujon) ou cryptique (chevaine qui manifeste une préférence écologique nette pour les habitats de berge). Mais toutes les espèces rhéophiles concentrées dans les milieux lotiques sont plus aisément capturèes que les espèces solitaires (brochet) ou grègaires (perche, gardon) qui affectionnent les habitats lentiques où l'efficacitè de la pêche diminue à cause de la profondeur.

\section{d) Densité des populations}

Toutes autres conditions égales, la probabilité de capturer $(=$ probabilité 
de contact) une espèce donnée est inversement proportionnelle à la densité du peup!ement spécifique. Cet élément contribue de manière décisive à la faible capturabilité relative du brochet, de la perche, du gardon, de la vandoise et de l'ombre dont les populations sont peu denses.

$\mathrm{Vu}$ les conditions de mesure des efficacites relatives de capture, il faut envisager l'interférence possible de phénomènes tels que :

a) perte de marque, b) migration des poissons marqués en dehors du secteur étudié, c) mortalité des poissons marqués suite aux manipulations subies, d) diminution de la vulnérabilité ultérieure des poissons capturès une première fois par pêche à l'électricité (LELEK, 1966; CROSS et STOTT, 1975). L'utilisation de marquages de groupe sur de courtes périodes et la réalisation des expériences de marquage-recapture dans un très long tronçon de rivière réduisaient très fortement les risques a) et b). II s'écoulait au moins un mois entre les pêches de marquage et de recapture et le risque d) pouvait raisonnablement être considéré comme négligeable.

Par contre, le risque c) aurait pu jouer avec des espèces assez fragiles (stress de la capture et des manipulations, perte d'écailles, déchirure des nageoires) comme la vandoise, le gardon, le hotu, l'ombre et la perche. Signalons cependant que dans les petites rivières cyprinicoles (moins de $10 \mathrm{~m}$ de largeur) où existent des conditions d'échantillonnage complet du milieu (facies lentique et lotique), on atteint avec le gardon et la vandoise par exemple des efficacités de capture aussi élevées que celles calculées pour le chevaine et le barbeau de l'Ourthe.

\subsection{Efficacité absolue en fonction du milieu}

\section{a) Influence de la profondeur (habitat)}

Pour une espèce ou un groupe subspécifique donné et dans des conditions physico-chimiques connues et constantes, l'efficacité absolue de la pêche avec un courant de forme determinée est directement limitée par la profondeur de l'eau et les paramètres associés (vitesse du courant, végétation); dans les rapides peu profonds à végétation aquatique bien développée, les captures sont relativement plus aisées, plus nombreuses et moins sélectives à l'égard de la tail!e que dans les zones profondes à courant lent et pauvres en végétation servant d'abri et de lieu de concentration. 


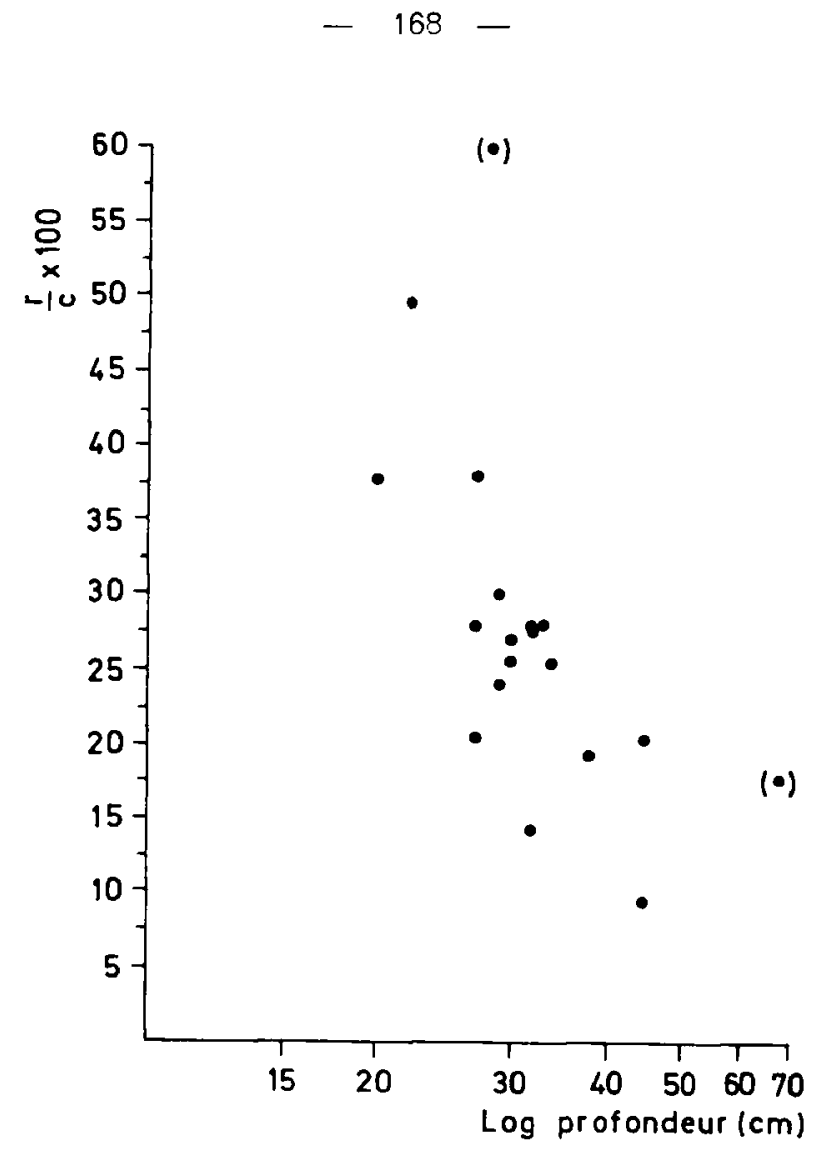

Fig. 5 : Relation entre l'efficacité de la pêche à l'électricité pour la capture du barbeau et la profondeur moyenne de la rivière. L'analyse concerne 19 sous-secteurs/habitats soumis à un dénombrement par marquage-recapture simple en août-septembre 1973. Les profondeurs moyennes sont celles de l'étiage 1975 (cf. fig. 2).

La comparaison des rapports $\mathrm{r} / \mathrm{c}$ dans 19 sous-secteurs/habitats montre une relation (fig. 5) entre l'efficacité de capture (mesurée par les rapports $\mathrm{r} / \mathrm{c}$ des barbeaux de plus de $20 \mathrm{~cm}$ ) et la profondeur moyenne de la rivière. L'accroissement de profondeur diminue de manière très significative la probabilité de capturer les barbeaux; le même phènomène s'observe chez la plupart des autres espèces rhéophiles. Les causes en sont diverses et agissent le plus souvent en synergie. Citons sans entrer dans les détails : non-accessibilité absolue en cas de grande profondeur $(>2 \mathrm{~m}$ ), possibilité de fuite accrue en milieu ouvert, difficulté à " récupérer "les poissons étourdis en raison du courant lent (nage forcée peu marquée) de la profondeur elle-même et du manque de visibilité dans l'eau et enfin probabilité de contact réduite du fait de densités de peuplement généralement inférieures à la moyenne.

\section{b) Influence du débit}

Un autre aspect important des problèmes d'échantillonnage est la variation temporelle (journalière, saisonnière, annuelle) de l'éfficacité de pêche. Nous avons calculé les rapports $\mathrm{r} / \mathrm{c}$ pour 6 échantillonnages intensifs réalisés en période estivale (juin, août, septembre 1972, 1973, 1974) dans un méme secteur de 
$1550 \mathrm{~m}$. L'analyse porte sur des séquences de marquage-recapture à court terme (juin-août et août-septembre) ce qui réduit les risques d'interférence, toujours pius grande à long terme, de la mortalité des marqués et des mouvements.

Pour les barbeaux mesurant plus de $20 \mathrm{~cm}$. l'efficacité de capture varie entre un minimum de 0,076 en septembre 1972 et un maximum de 0,194 en août 1973. Les expériences ayant été réalisées dans les conditions de stabilité maximaje des poissons, ces différences s'expliquent par une influence du débit de la rivière (fig 6). L'influence négative du débit sur l'efficacité de pêche provient d'un effet purement mécanique (augmentation de la profondeur et accélération du courant) d'un effet chimique (réduction de la conductivité électrique par dilution : $350 \mu \mathrm{S} / \mathrm{cm}$ à l'étiage, $150 \mu \mathrm{S} / \mathrm{cm}$ en période de crue) et d'un effet lié à la turbidité de l'eau (difficulté de localiser les poissons).

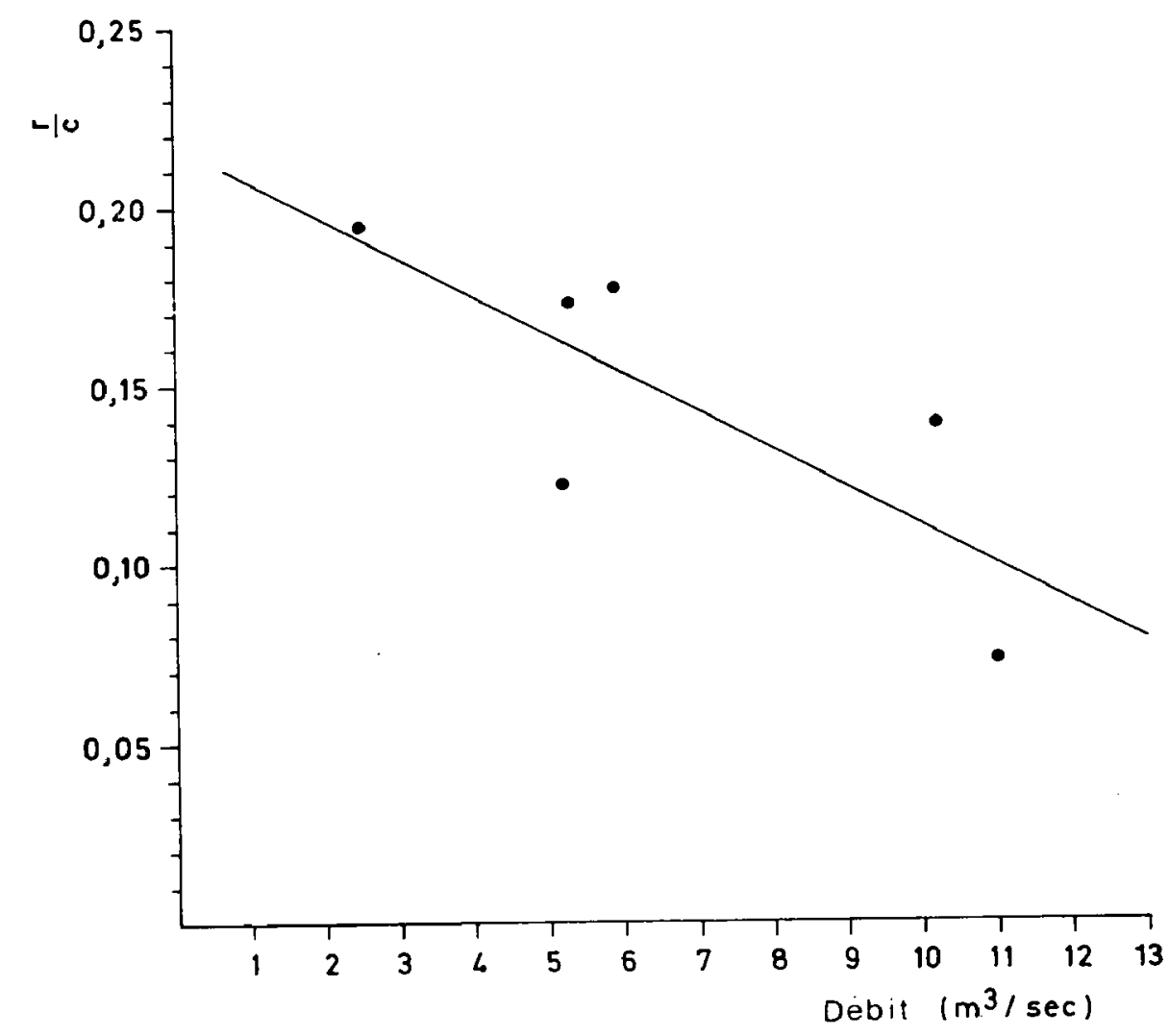

Fig. 6 : Relation entre l'efficacité de la pêche à l'électricité pour la capture des barbeaux et le débit moyen de l'Ourthe pendant la période de marquagerecapture. L'analyse porte sur 6 séquences de pêche intensive réalisées de 1972 à 1974 dans un secteur de $1550 \mathrm{~m}$.

\section{c) Influence d'autres facteurs}

La température de l'eau, non envisagée dans cette étude, influence l'efficacité de la pêche à l'électricité 
- indirectement. en contrôlant l'activité saisonnière des poissons (par exemple, hivernage en groupe dans les endroits profonds et inaccessibles) ou via la liaison température-débit-hauteur d'eau ou turbidité,

- directement, en modifiant la réactivité au courant électrique.

L'élévation de la température augmente le métabolisme des poissons et leur conductivité corporelle; il en résulte une plus grande excitabilité (seuil de sensibilité plus bas) mais en même temps, une plus grande résistance à la galvanonarcose; ces deux phénomènes observès chez la truite (HALSBAND, 1967 ; VINCENT, 1971) et le chevaine (PENAZ et PROKES, 1973), se traduisent par une diminution de la vulnèrabilité (fuite plus rapide à l'approche des pêcheurs et moins bonne réaction lors du contact direct) (cf. aussi LAMARQUE et coll., 1975).

Parmi les autres influences possibles, signalons encore celles de divers facteurs physiques du milieu, en pratique difficilement quantifiables : présence de caches, de branches, de rochers, etc... qui concentrent les poissons et facilitent leur capture dans les zones profondes, développement de la végétation aquatique qui joue le même rôle en facies lotique, notamment en freinant la fuite du poisson devant l'équipe de pêche.

Restent enfin les facteurs humains (habitude, fatigue, etc...) souvent très importants mais dont l'interférence ètait considérablement réduite dans notre cas par l'utilisation d'une même équipe de 5 personnes, ayant une bonne connaissance de la technique de pêche et de la rivière.

\section{Conclusions}

Les variations interspécifiques, intraspécifiques (sélectivité selon la taille), spatiales (habitats - biotopes) et temporelles (jour, saison) de l'efficacité de la pêche à l'électricité sont des élèments essentiels à considérer pour mettre au point une stratégie d'échantillonnage quantitatif et de dénombrement statistique des populations et communautés de poissons (cf. LAURENT, 1978). Ce problème est particulièrement important dans les rivières du type «Zone à Barbeau», à cause de la diversité des espèces et de l'hétérogénéité du milieu.

Au niveau de la communauté ichtyologique, il s'agit d'abord de capturer un nombre suffisant de poissons de chaque espèce de manière à calculer des estimations fiables des peuplements. En fonction de leur vulnèrabilitè à la pêche à l'électricitè, quatre groupes d'espèces se dégagent, pour lesquelles l'optimalisation des captures est obtenue en utilisant des modalités de pêche particulières :

Groupe 1 : Truite, barbeau, chevaine: espèces localisèes dans les courants ou le long des berges (chevaine surtout). Pêche à pied de l'aval vers l'amont dans les radiers et les plats ou pêche en barque le long des berges, dans les calmes.

Groupe 2 : Hotu, vandoise, ombre: espèces rhéophiles plus ou moins pélagiques. Pêche à pied de l'amont vers l'aval ou pêche en barque en descente libre dans les plats et les calmes (effet de surprise).

Groupe 3 : Brochet, gardon, perche: espèces limnophiles. Pêche en barque dans les calmes (en pleine eau et le long des berges) complétée par d'au. tres méthodes : senne, senne èlectrifièe, pièges, filets maillants. 
Groupe 4 : Goujon, ablette de rivière, vairon, chabot, loche : poissons de petite taille exigeant un échantillonnage intensif sur de faibles surfaces.

Au niveau des populations spécifiques, l'effet de la sélectivité de la pêche selon la taille est aisément éliminè en calculant des estimations séparées pour différentes catégories de longueur.

L'influence des facteurs débit-turbidité-température peut être réduite en opérant les échantillonnages en conditions hydrologiques constantes, ce qui est difficilement i réalisable en pratique lors de séquences de pèches sur de longs tronçons ide rivière.

Enfin, les variations de l'efficacité de la pêche selon les habitats (profondeur) restent un problème majeur lorsque des contraintes biologiques (dimension du domaine vital) imposent des dénombrements par marquage-recapture sur de longs secteurs (plus de $1000 \mathrm{~m}$ ), forcément hétérogènes. Cet aspect de la question est analysé dans une autre publication consacrée au barbeau.

\section{BIBLIOGRAPHIE}

CROSS D.G. et STOTT B., 1975. The effect of electric fishing on the subsequent capture of fish. J. Fish. Biol., 7, 349-357.

HALSBAND E., 1967. Principes fondamentaux de la pêche à l'électricité, p. 79-85, in VIBERT, R., Application de l'électricité à la biologie et à l'aménagement des pêches continentales. Symp. CECPI, Belgrade, 1966, INRA - Paris.

HUET M., 1949. Aperçu des relations entre la pente et les populations piscicoles des eaux courantes. Schweiz. Z. Hydrol., 11 (3-4) : 332-351.

LAMARQUE P., THEREZIEN Y. et CHARLON N., 1975. Etude des conditions de la pêche à l'électricité dans les eaux tropicales. Bull. Cent. Etud. Rech. Sci., Biarritz, 10 (3) : 403-554.

LAURENT M. et LAMARQUE P., 1974. Utilisation de la méthode des captures successives (Delury pour l'évaluation des peuplements piscicoles. Ann. Hydrobiol., 5 (2) : 121-132.

LAURENT M., 1978. Etude des erreurs d'estimation des populations par la méthode des captures successives (DeLury, 2 captures) et des captures-recaptures (Petersen). Bull. Fr. Pisc., no $268: 162-172$.

LELEK A., 1966. The field experiment on the receiptivity of chub, Leucisclis cephalus (L.) to the repeated effect of pulsating direct current. Verh. Internat. Verein. Limnol., 16 : 1217-1222.

MICHA J.C et PIUWET J.C., 1970. La pêche électrique en rivière et ses applications dans la région liégeoise. Naturalistes Belges, 51 (6) : 291-306.

MICHA J.C., 1971. Etude des communautés piscicoles dans l'Ourihe liégeoise Tribune du CEBEDEAU, no $326: 1-8$.

MICHA J.C. et DE MOFFAERTS E., 1975. Les poissons de la Sambre belge. Modèles mathématiques de la Sambre. Rapport de synthase. Annexe Ili, Aspects biologiques de la Sambre (partie 1). Commission Interministerielie de la Politique Scientifique. 
PENAZ M. et PROKES M., 1973. Response of the chub, Leuciscus cephalus in a homogeneous field of continuous and interrupted D.C. Zool. listy, 22 (3) : 265-282.

PHILIPPART J.C., 1977a. Contribution à l'étude de l'écosystème "Rivière de la Zone à Barbeau supérieure ": Densité, biomasse et production des populations de poissons de l'Ourthe: pp. 551-567, in DUVIGNEAUD, $P$. et $P$. KESTEMONT (Ed.), Productivité biologique en Belgique,, Gembloux Paris : $617 \mathrm{p}$.

PHILIPPART J.C., 1977b. Contribution à l'hydrobiologie de l'Ourthe. Dynamique des populations et production de quatre especes de poissons cyprinidae : Barbus barbus (L.), Chondrostoma nasus (L.), Leuciscus cephalus (L.) et Leuciscus leuciscus (L.). Thèse de Doctorat en Sc Zoologiques. Univ. Liège : $225 \mathrm{p}$.

SEBER G. A.F., 1973. The estimation of animal abundance and related parameters. Griffin London, 506 pages.

VINCENT R., 1971. River electrofishing ant fish population estimates. Prog. Fish. Cult., 33 (3): 163-169. 